\title{
Modeling whistler wave generation regimes in magnetospheric cyclotron maser
}

\author{
D. L. Pasmanik ${ }^{1}$, A. G. Demekhov ${ }^{1}$, V. Y. Trakhtengerts ${ }^{1}$, and M. Parrot ${ }^{2}$ \\ ${ }^{1}$ Institute of Applied Physics RAS, Ulyanov Street 46, Nizhny Novgorod 603950, Russia \\ ${ }^{2}$ LPCE/CNRS, Orléans, France
}

Received: 19 March 2003 - Revised: 30 March 2004 - Accepted: 18 May 2004 - Published: 3 November 2004

\begin{abstract}
Numerical analysis of the model for cyclotron instability in the Earth's magnetosphere is performed. This model, based on the self-consistent set of equations of quasilinear plasma theory, describes different regimes of wave generation and related energetic particle precipitation. As the source of free energy the injection of energetic electrons with transverse anisotropic distribution function to the interaction region is considered. A parametric study of the model is performed. The main attention is paid to the analysis of generation regimes for different characteristics of energetic electron source, such as the shape of pitch angle distributions and its intensity. Two mechanisms of removal of energetic electrons from a generation region are considered, one is due to the particle precipitation through the loss cone and another one is related to the magnetic drift of energetic particles.

It was confirmed that two main regimes occur in this system in the presence of a constant particle source, in the case of precipitation losses. At small source intensity relaxation oscillations were found, whose parameters are in good agreement with simplified analytical theory developed earlier. At a larger source intensity, transition to a periodic generation occurs. In the case of drift losses the regime of self-sustained periodic generation regime is realized for source intensity higher than some threshold. The dependencies of repetition period and dynamic spectrum shape on the source parameters were studied in detail. In addition to simple periodic regimes, those with more complex spectral forms were found. In particular, alteration of spikes with different spectral shape can take place. It was also shown that quasi-stationary generation at the low-frequency band can coexist with periodic modulation at higher frequencies.
\end{abstract}

On the basis of the results obtained, the model for explanation of quasi-periodic whistler wave emissions is verified.

Key words. Magnetospheric physics (plasma waves and instabilities; energetic particles, precipitating; energetic particles, trapped)

Correspondence to: D. L. Pasmanik

(pdl@aurora.appl.sci-nnov.ru)

\section{Introduction}

Quasi-periodic (QP) whistler emissions are the wide band emissions that are observed inside or near the plasmapause (see, for example, Helliwell (1965); Sato et al. (1974); Hayakawa and Sazhin (1992); Sazhin and Hayakawa (1994)). They are characterized by a periodic modulation of wave intensity with typical periods from several seconds up to a few minutes. Generation of QP emissions is usually accompanied by precipitation of energetic particles, which are also modulated by the same period.

One of the possible mechanisms of such periodic modulation is connected to geomagnetic pulsations of a corresponding period (Sato et al., 1974). But experimental data show that generation of QP emissions may occur in the absence of such disturbances (Oguti et al., 1991). It is believed that in the latter case, whistler waves are generated by the cyclotron instability in a region with enhanced cold plasma density. The free energy for wave excitation is supplied to the interaction region by the energetic electrons with transverse anisotropic distribution function. The source of these particles may be related to their magnetic drift to the interaction region or to a local acceleration mechanism, such as the magnetic tube compression.

Particles can be removed from the interaction region by two mechanisms: precipitation of energetic particles via the loss cone and drift of particles away from the interaction region across the magnetic field lines.

Drift removal of energetic particles is most effective for interaction in a duct that is outside the plasmasphere or if the cross section of the interaction region is rather small. This mechanism was taken into account in the flow cyclotron maser (FCM) model developed by Demekhov and Trakhtengerts (1994). Periodic regimes of whistler wave generation obtained in this model were used for explanation of pulsating auroras.

In the case of interaction inside the plasmasphere or in rather large areas of enhanced cold plasma density, losses of energetic particles due to precipitation are dominant. Such a mechanism was considered in a simplified, self-consistent 
model of cyclotron interaction by Bespalov and Trakhtengerts (1976) and Davidson (1979), where the so-called two-level approximation was used. The results obtained on the basis of this model allow one to explain general properties and characteristic time scales of whistler wave generation, but this model does not take into account the evolution of wave spectrum and energetic particle distribution.

In this paper, we present results of numerical analysis of both cases mentioned above. The FCM model is generalized with loss cone precipitation taken into account. We performed extensive numerical analysis of this model using various combinations of parameters for both removal mechanisms to find which generation regimes are possible in such a system. We analyze the properties of these generation regimes as dependent on the system parameters, such as characteristics of the energetic particle source and wave damping. Peculiarities of the wave spectra corresponding to each generation regime are discussed. The results obtained should be useful for interpretation of many available experimental data for QP emissions.

\section{Description of the model}

As it was mentioned above, we assume in our model that QP emissions are generated in the region with enhanced cold plasma density, where the cyclotron resonance condition is satisfied for most of the energetic particles (see below). Outside this region, the development of cyclotron instability is impossible and the distribution of energetic electrons may remain anisotropic. The magnetic drift of such particles into the region with dense plasma serves as the source of free energy for wave generation.

To describe the cyclotron instability development in this region we use the self-consistent set of equations of quasilinear plasma theory for the distribution function $F(\mu, v, t)$ of energetic electrons and whistler wave spectral energy density $\varepsilon(\omega, t)$. To study processes with characteristic timescales greater than periods of oscillations of waves and particles between mirror points (which is true for QP emissions), it is possible to use equations averaged over these oscillations (Bespalov and Trakhtengerts, 1986). Below we shall consider only a low-frequency band $\omega \ll \omega_{B}$, where $\omega_{B}$ is the electron gyrofrequency. In this case pitch angle diffusion dominates over the energy diffusion and equations of quasilinear theory can be written in the following form (Bespalov and Trakhtengerts, 1986):

$$
\begin{aligned}
& \frac{\partial F}{\partial t}+V_{D} \frac{\partial F}{\partial x}=\frac{1}{T_{b}} \frac{\partial}{\partial \mu} \mu D \frac{\partial F}{\partial \mu}-\delta \cdot F \\
& \frac{\partial \varepsilon}{\partial t}=\frac{2}{T_{g}}(\Gamma-|\ln R|) \varepsilon .
\end{aligned}
$$

Here $\mu=\sin ^{2} \Theta_{L}, \Theta_{L}$ is the equatorial pitch angle, $v$ is the electron velocity, $T_{b}$ is the bounce oscillations period, $T_{g}$ is the period of wave-packet oscillations, and $R$ is the effective reflection coefficient, describing wave energy loss. The coefficient $D$ of pitch angle diffusion and the amplification $\Gamma$ of whistler waves on the pass between conjugate ionospheres are defined below.

The second term on the left-hand side of Eq. (1), where $V_{D}$ is the velocity of magnetic drift, and $x$ is the coordinate across the magnetic field lines, describes the drift of energetic particles. Energetic particle losses due to their precipitation via the loss cone are taken into account by the last term in Eq. (1), where

$\delta= \begin{cases}0 & \mu \geq \mu_{c} \\ \left(T_{b} / 4\right)^{-1} & 0 \leq \mu \leq \mu_{c}\end{cases}$

and $\mu_{c}$ is the loss cone boundary.

In this paper we restrict our consideration to the case of ducted whistler wave propagation, in which the waves propagate parallel to the geomagnetic field (i.e. $\boldsymbol{k} \| \boldsymbol{B}$ ). Taking into account oblique electromagnetic whistler waves would not change the obtained results quantitatively. Direction finding studies confirm that whistler waves propagating at small angles in the near-equatorial region are typical in the near plasmapause region (see Hayakawa et al. (1986); Hayakawa and Sazhin (1992)). Quasi-electrostatic or unducted electromagnetic whistlers require a more complicated analysis, which is beyond the scope of this paper. In the case of longitudinal wave propagation, we can assume quasihomogeneous distribution of the wave energy $\varepsilon$ across the interaction region and average the kinetic Eq. (1) over the cross section of this region:

$$
\begin{aligned}
& \frac{\partial \Phi}{\partial t}=\frac{1}{T_{b}} \frac{\partial}{\partial \mu} \mu D \frac{\partial \Phi}{\partial \mu}+J-\delta \cdot \Phi \\
& J=\left(V_{D} / S_{0}\right) \int_{y_{1}}^{y_{2}}\left(F_{\text {in }}-F_{\text {out }}\right) d y,
\end{aligned}
$$

where $\Phi=S_{0}^{-1} \int F d s, S_{0}$ is the area of the duct cross section, $J$ is the effective source, $F_{\text {in }}$ and $F_{\text {out }}$ are the distribution functions of electrons entering and leaving the duct, respectively, and $y_{1}$ and $y_{2}$ are the boundaries of the interaction region in the direction transverse to $\boldsymbol{V}_{\boldsymbol{D}}$.

Taking into account that the energy diffusion is negligible in the considered frequency range $\omega \ll \omega_{B}$, we can simplify the analysis of this model by using a distribution with a narrow energy spectrum, parameterized by the characteristic energy $W_{0}=m v_{0}^{2} / 2$. The use of this approximation is possible due to the fact that the inhomogeneous geomagnetic field provides a spread in resonant energies: a wave with a given frequency $\omega$ interacts with particles whose velosity is $v>v_{\min }(\omega, \mu)$, where $v_{\min }$ is determined from the cyclotron resonance condition in the equatorial plane (see Appendix and the integration limits in Eqs. (6) and (7) below). Due to that, the growth rate of ducted whistler waves is determined by integral parameters of the energy distribution, such as characteristic energy (Bespalov and Trakhtengerts, 1976; Demekhov and Trakhtengerts, 1994). This approximation allows us to obtain correct integral characteristics, such as the 
particle flux and the wave amplification without analyzing the evolution of the particle energy spectrum. Hereafter, $\Phi$ will denotes the distribution function over $\mu$, i.e. the distribution integrated over its narrow energy spread near $W_{0}$.

Applying these simplifications to the full expressions for the diffusion coefficient and the wave amplification (Bespalov and Trakhtengerts, 1986), we obtain the following expressions (Demekhov and Trakhtengerts, 1994):

$D=D_{*} \cdot(2-\mu)^{-1 / 2} \int_{\mu}^{1} \varepsilon(\xi-\mu)^{-1 / 2} d \xi$

$\Gamma=\Gamma_{*} \cdot \frac{1-\xi}{\sqrt{2-\xi}} \int_{0}^{\xi}\left(\mu \frac{\partial \Phi}{\partial \mu}-\frac{\omega}{\omega_{B L}} \Phi\right)(\xi-\mu)^{-1 / 2} d \mu$,

where

$D_{*}=\frac{32 \sqrt{2} \pi^{2} e^{2} L R_{0}}{3 m^{2} c^{2} v_{0} \beta_{*}}, \quad \Gamma_{*}=\frac{\pi a \omega_{p L}^{2} v_{0}}{\omega_{B L} c^{2} n_{c L}}$

$\xi=1-\omega_{0} / \omega, \beta_{*}=\left(\frac{\omega_{p L} v_{0}}{\omega_{B L} c}\right)^{2}, \omega_{0}=\omega_{B L} / \beta_{*}$,

where $\omega_{B}$ and $\omega_{p}$ are the electron gyrofrequency and plasma frequency, respectively, the subscript $L$ refers to the values in the equatorial plane, $e$ and $m$ are the electron charge and mass, and $c$ is the velocity of light in free space. The parameter $\beta_{*}$, proportional to the cold plasma density and characteristic particle energy, determines which fraction of particles are in resonance: the number of resonant particles increases with an increase in $\beta_{*}$, and for $\beta_{*} \gg 1$ almost all particles are resonant; the value $\omega_{0}$ corresponds to the lowest frequency of whistler waves interacting with electrons having the energy $W_{0}$. The parabolic approximation for both the magnetic field $B$ and cold plasma density distribution $n_{c}$ along the geomagnetic field line near the equatorial region was used, i.e. the proportionality $n_{c} \propto B$ was used:

$$
\frac{B}{B_{L}}=\frac{n_{c}}{n_{c L}}=\left(1+\frac{z^{2}}{a^{2}}\right),
$$

where $z$ is the coordinate along the magnetic field line, $a=\sqrt{2} R_{0} L / 3$ is the characteristic scale of the Earth's magnetic field at the magnetic shell $L$, and $R_{0}$ is the Earth's radius.

A more detailed explanation of how the expressions for wave amplification (7) and diffusion coefficient (6), obtained from the full formulation of Bespalov and Trakhtengerts (1986), is given in the Appendix.

\section{Numerical results}

In this section, we present the results of numerical analysis of the model described above. The main attention was paid to study the dependence of the generation regime on the properties of the energetic particle source (i.e. characteristics of the distribution $F_{\text {in }}$ of drifting particles), such as the shape and amplitude of pitch angle distribution, and on the frequency dependence of the wave damping $R(\omega)$.

The following values for other parameters were used in our simulations: $L=4.4\left(f_{B L} \approx 10.3 \mathrm{kHz}\right), n_{c L}=55 \mathrm{~cm}^{-3}$, and $W_{0}=45 \mathrm{keV}$.

As it was mentioned above, two different mechanisms of energetic particle removal from the generation region are taken into account in the kinetic equation (4). In this paper we analyse these mechanisms separately, i.e. two cases are studied: (1) where only losses due to precipitation are significant (in this case $F_{\text {out }} \equiv 0$ ), and (2) where only drift losses are taken into account (in this case $\delta \equiv 0$ ).

\subsection{The case of losses due to precipitation}

We performed extensive numerical analysis of this case using various combinations of parameters. According to results obtained, the crucial parameter determining the generation regime in the system is the intensity of source, i.e. the number of particles supplied in time unit. A variation in other characteristics of the particle source and wave damping affect the generation regime much lesser. Thus, for this case we discuss only the dependence of generation regime on the source intensity. In particular examples presented below, the source with moderate pitch angle anisotropy was used:

$F_{\text {in }}(\mu)=C \sqrt{\mu}$,

where the value $C$ is defined by the normalization condition

$\int_{0}^{1} F_{\text {in }} \frac{d \mu}{\sqrt{1-\mu}}=n_{0}$

and $n_{0}$ is energetic electron density. It is convenient to characterize the intensity of the source by the value

$J_{0}=v_{0} \int_{0}^{1} F_{\text {in }} d \mu$.

It is equal to the flux of precipitating electrons provided by the source in the stationary state (i.e. when the number of precipitating particles is equal to that supplied by the source).

As a dimensionless characteristic of the source intensity, one can use the value $j_{0}=\left(v \tau_{J}\right)^{-1}$, where $\tau_{J}=N / J_{0}$ is the time scale of energetic particle supply, $N$ is the number of energetic electrons in the magnetic flux tube, and $\nu=2|\ln R| / T_{g}$ is the wave damping rate.

In the case of a weak source, the regime of relaxation oscillations in wave generation takes place. In this regime, after the system reaches the threshold of the cyclotron instability, several spikes in the wave intensity with diminishing amplitude are generated. After that, the system goes into the stationary generation regime. An example of such a generation regime is presented in Fig. 1. The wave spectrogram is shown on the left panel, and the snapshots of electron distribution function corresponding to the minimum and maximum wave intensity are shown on the right panel. 

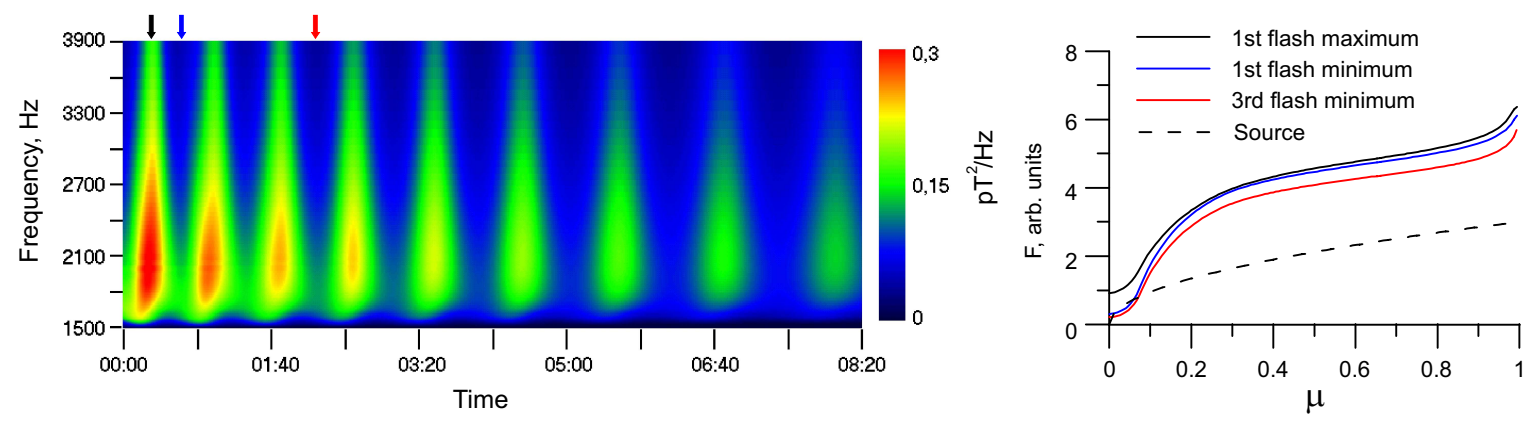

Fig. 1. Relaxation oscillations regime in the model with losses due to precipitation. Spectrogram of the wave intensity is on the left panel and snapshots of pitch angle distribution of energetic electrons in the duct (solid lines) and distribution in the source defined by Eq. (11) (dashed line) are on the right panel, time marks for snapshots are given in the corresponding color at the top of the spectrogram. System parameters are $L=4.4, n_{c L}=55 \mathrm{~cm}^{-3}, W_{0}=45 \mathrm{keV}, \ln R=2 \omega / \omega_{0}, J_{0}=10^{6} \mathrm{~cm}^{-2} \mathrm{~s}^{-1}$.

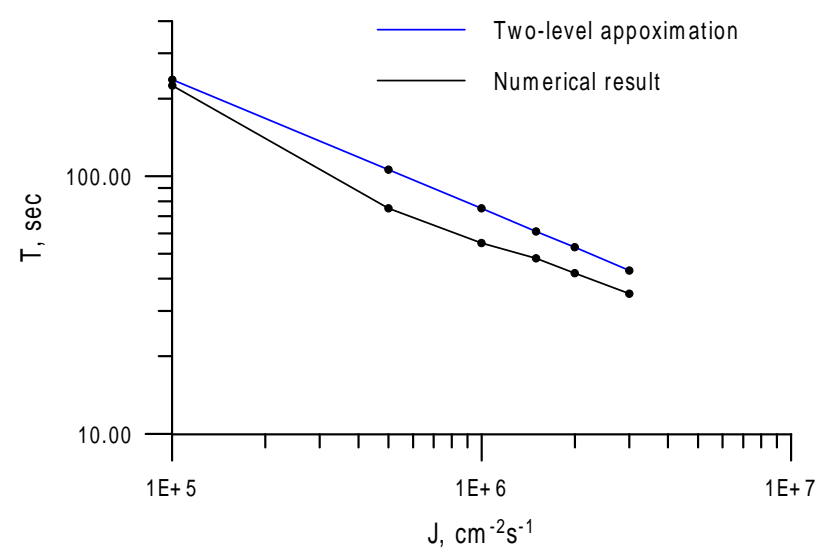

Fig. 2. Dependence of period of relaxation oscillations on the intensity of the energetic particle source. Results from simplified "twolevel approximation" model and from numerical analysis of the full system Eqs. (1) and (2) are presented. The same system parameter values (except the source intensity) as in Fig. 1 were used.

As one can see, this generation regime is characterized by almost constant shapes of the wave spectrum and electron pitch angle distribution, whose amplitudes change in time. With an increase in the source intensity, both the oscillation period and the characteristic relaxation time decrease.

A simplified analytical model for such a generation regime has been developed by Bespalov and Trakhtengerts (1976) (see also the review Bespalov and Trakhtengerts, 1986). To obtain this model, the so-called two-level approximation is used, where the shapes of wave spectrum and electron pitch angle distribution remain constant in the process of cyclotron instability development. This assumption allows one to obtain from Eq. (1) the system of ordinary differential equations for new variables: the number $N$ of energetic electrons in the magnetic flux tube and the wave energy density $E$. Analysis of this simplified model yields the following expression for the period of relaxation oscillations:

$T=2 \pi \sqrt{\frac{N_{0} / J_{0}}{\ln R / T_{g}}}$, where $N_{0}$ is the average number of trapped energetic electrons in the magnetic flux tube with a unit cross section at the ionospheric level. We performed a comparison of this result from the simplified model with the results obtained from the numerical solution of the full system Eqs. (1)-(2). As one can see from Fig. 2 these two approaches are in good agreement.

With further increase in the energetic particle source intensity, another generation regime occurs - the regime of selfsustained oscillations. In this regime, periodic undamped oscillations of wave intensity and precipitating particle flux take place (Fig. 3). An important feature of this regime is a positive frequency drift during a single spike in the wave intensity (see Fig. 3, the left panel). This happens because new particles with higher values of the pitch angles become involved in the interaction as the cyclotron instability develops. This is also seen in a different time evolution of the energetic electron distribution function as compared to relaxation oscillations: one can note stronger variation in the pitch angle distribution shape from the maximum to the minimum of wave intensity (Fig. 3, the right panel).

An increase in the source intensity leads to a decrease in the oscillation period, but there is no more qualitative changes in the generation regime. On the whole, generation regimes in the case of precipitation losses are characterized by rather small modulation of wave intensity and small variation in the energetic electron pitch-angle distribution.

\subsection{The case of drift losses}

As it was mentioned above, this case was studied by Demekhov and Trakhtengerts (1994) as an application to pulsating aurora phenomenon (FCM model). The main attention was paid to the analysis of temporal parameters of the generation regime (such as spike duration and repetition period), and mainly integral characteristics of the system were considered (such as wave energy density). Dependence of these parameters on the plasma properties (magnetic shell, cold plasma density) was studied. In this paper, we focus on analysis of how the dynamics of wave spectra and electron 

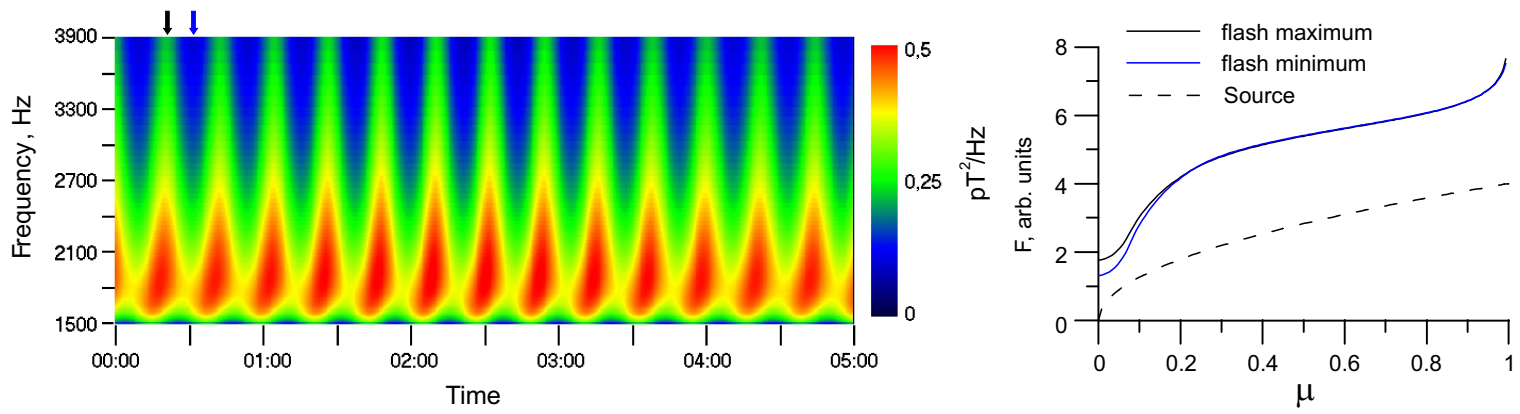

Fig. 3. Regime of self-sustained oscillations in the case of losses due to precipitation. The source of energetic particles is 10 times greater then in Fig. $1\left(J_{0}=10^{7} \mathrm{~cm}^{-2} \mathrm{~s}^{-1}\right)$, all other parameters and time marks legend are the same as in Fig. 1.
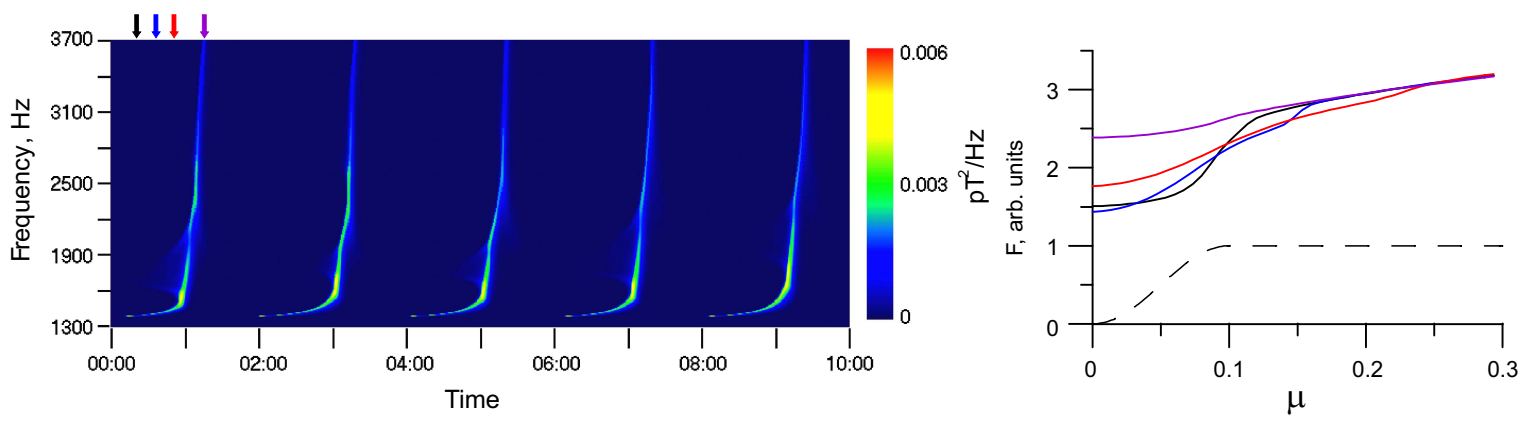

Fig. 4. Periodic generation of wave intensity spikes in the case of losses due to drift and monotonic pitch angle distribution in the source Eq. (18). System parameters are $L=4.4, n_{c L}=55 \mathrm{~cm}^{-3}, W_{0}=45 \mathrm{keV}, \ln R=2 \omega / \omega_{0}$, the density of drifting energetic particles $n_{h L}=0.04 \mathrm{~cm}^{-3}$, the duct radius in the equatorial plane $100 \mathrm{~km}$; source parameters are $\mathfrak{x}=0.1, \delta=2$. The same time marks legend as in Fig. 1 is used. The pitch angle distribution in the source is shown by the dashed line.

pitch angle distribution depend on the properties of the energetic particle source.

To solve Eq. (4) for the distribution function $\Phi$ it is necessary to know the distribution $F_{\text {out }}$ of energetic particles at the exit from the duct. The shape of this distribution is determined by the evolution of initial distribution $F_{\text {in }}$ during particle drift across the duct and depends on the dynamics of cyclotron instability development in the duct. Following Demekhov and Trakhtengerts (1994), we assume that the distribution $F_{\text {out }}$ is isotropic at all $\mu$ and its amplitude is defined from the particle conservation law:

$$
\int F_{\text {out }} T_{b} d \mu=\int F_{\text {in }} T_{b} d \mu
$$

Such an assumption can be justified if the loss cone is small enough, the wave intensity is rather high, and the main free energy source is due to the pitch angle anisotropy of trapped particles.

Wave generation in this case is possible if the intensity of the source is higher than some threshold value, which is determined from the condition that the cyclotron instability threshold is exceeded if the whole duct is filled by energetic electrons:

$\Gamma_{0} \geq \ln R$, where the value of $\Gamma_{0}$ is calculated from Eq. (7) using the distribution $F_{\text {in }}$. This is different from the case of a large interaction region (Sect. 3.1), in which the instability threshold may be reached due to constant accumulation of energetic particles.

In contrast to the previous case, much wider variety of generation regimes exists in the case of drift losses, and the dependence of their characteristics on the system parameters is much stronger. In particular, the shape of the source (the initial pitch angle distribution of drifting electrons) is one of the crucial parameters determining the generation regime. Three different types of pitch angle distribution in the source are studied here. The first type corresponds to the monotonic pitch angle distribution with moderate anisotropy:

$F_{\text {in }}=C \mu^{\alpha}$,

where the parameter $\alpha$ characterizes the anisotropy. Our analysis showed that in this case the generation regimes are rather similar to those discussed in the previous section: stationary generation, relaxation oscillations, and self-sustained oscillations with small modulation of wave intensity. Thus we refer the reader to the Sect. 3.1 for a discussion of these regimes. 

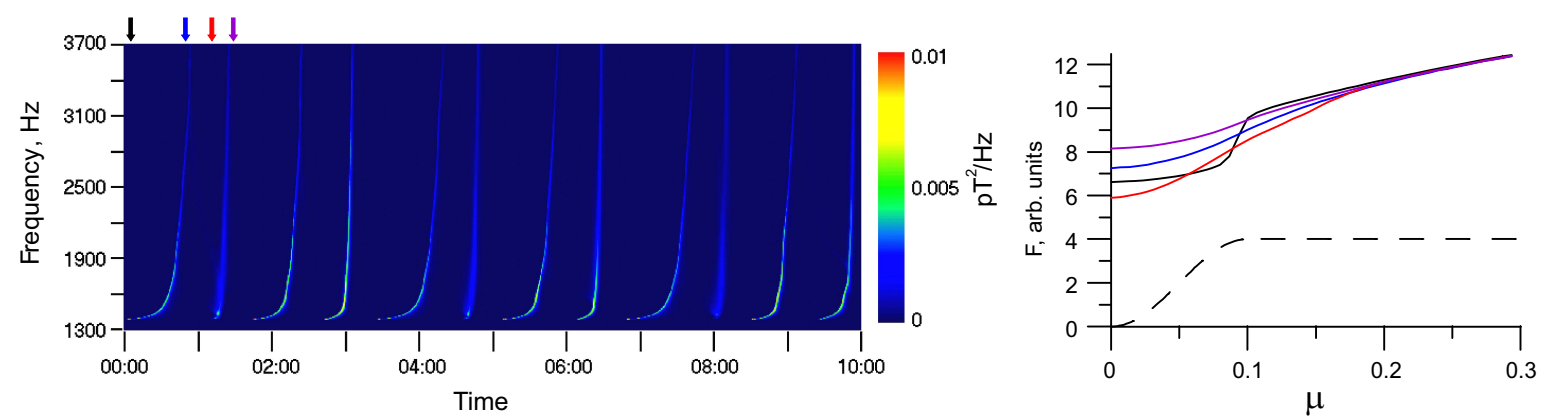

Fig. 5. Periodic generation of wave intensity spikes in the case of losses due to drift with intermission of spikes of two different shapes. The source of energetic particles is 4 times greater than in Fig. $4\left(n_{h L}=0.16 \mathrm{~cm}^{-3}\right)$; all other parameters are the same.

The second type of the source has the form (see Fig. 4, the right panel)

$F_{\text {in }}=\left\{\begin{array}{ll}C \sin ^{\delta}\left(\frac{\pi}{2} \mathfrak{x} / \mathfrak{x}_{0}\right), & \mathfrak{x} \leq \mathfrak{x}_{0} \\ C, & \mathfrak{x}>\mathfrak{x}_{0}\end{array}\right.$,

where $æ \equiv \sqrt{\mu}$. This distribution can model, for example, the loss cone distribution if we put $\mathfrak{x}_{0} \approx \sqrt{\mu_{c}}$, or any other anisotropic source. The parameter $\delta$ characterizes the sharpness of the slope of the distribution.

A typical spectrogram for low intensity of the source (but still high enough to satisfy Eq. (16)) is shown in Fig. 4. This is the regime with periodic generation of wave intensity spikes. This regime is characterized by rather high modulation of wave intensity and by excitation of relatively narrow band waves in comparison with the regimes discussed above. Generation of a spike is accompanied by a shift in the maximum wave amplitude to higher frequencies. Such generation occurs due to formation of rather sharp gradient in the energetic electron pitch angle distribution (Fig. 4, the right panel) at the boundary between resonant and non-resonant particles, which moves toward higher pitch angles as the cyclotron instability develops (Demekhov and Trakhtengerts, 1994).

The duration of a spike is mainly determined by the pitch angle distribution of the source and is of the order of the inverse initial growth rate $\left(2 \ln R / T_{g}\right)^{-1}$. The interval between spikes is determined by the source intensity and is equal to the time needed to supply enough energetic particles with anisotropic distribution to satisfy condition Eq. (16). Accordingly, the period of spike generation decreases with increase in the source intensity. This is seen from Fig. 5, where the intensity of the source is 4 times higher than in Fig. 4.

Another interesting feature that appears with an increase in the source intensity and is seen in Fig. 5 is the intermission of spikes with two different shapes - one is similar to the case of a weak source (Fig. 4), and the second has a shape with much faster frequency shift but lower wave amplitude. It is also seen that generation of a spike of the second type starts immediately after the fade of the previous one, but there is a pronounced gap between the pairs of spikes. The corresponding evolution of energetic electron distribution is shown in Fig. 5, the right panel: the sharp gradient exists during generation of the first spike and is absent during generation of the second spike.

The existence of such a generation regime is explained as follows. When the intensity of the source is rather small, its contribution during the generation of a single spike is negligible. With an increase in the source intensity, the sourcerelated modification of the distribution of energetic electrons during spike generation becomes more significant. This leads to an increase in the distribution anisotropy at low $\left(\mathfrak{x} \sim \mathfrak{x}_{0}\right)$ pitch angles, but the pitch angle diffusion due to waves generated by a sharp gradient at higher æ values does not allow the system to reach the instability threshold at lower frequencies. As a result of joint action of the source and diffusion, at the end of the first spike the system comes to the state close to the threshold at all frequencies and with rather smooth pitch angle distribution (Fig. 5, the right panel). Thus, after the end of the first spike a rather small addition of anisotropic electrons by the source is required to initiate the generation of the next spike. According to that said above, simultaneous generation in a wide frequency band occurs, resulting in a different spectral shape of the second spike. Smaller intensity of this spike is explained by a smaller amount of free energy accumulated during a shorter time interval before the spike and by a wider distribution of the wave spectral energy in the spike. Generation of this short second spike results in an additional release of free energy after which the system returns to the initial state below the threshold.

Variation in other source parameters (i.e. the values of $æ$ and $\delta$ ) does not change the generation regime qualitatively. An increase in either $æ$ or $\delta$ leads to a decrease in the generation period and to an increase of the lowest wave frequency in a spike. This occurs because for the same source intensity ( $n_{0}$ value), such a change in the source parameters results in an increasing position $\mu_{\mathrm{opt}}$ and a value of the maximum of $\mu \partial F_{\text {in }} / \partial \mu$, which actually determine at which frequency and how fast the generation threshold Eq. (16) is exceeded.

The dependence of the generation characteristics on the wave damping frequency profile $R(\omega)$ was studied. It should be noted that the actual dependence $R(\omega)$, which is determined by reflection properties at the magnetic tube ends and by properties of wave propagation along the magnetic field is 

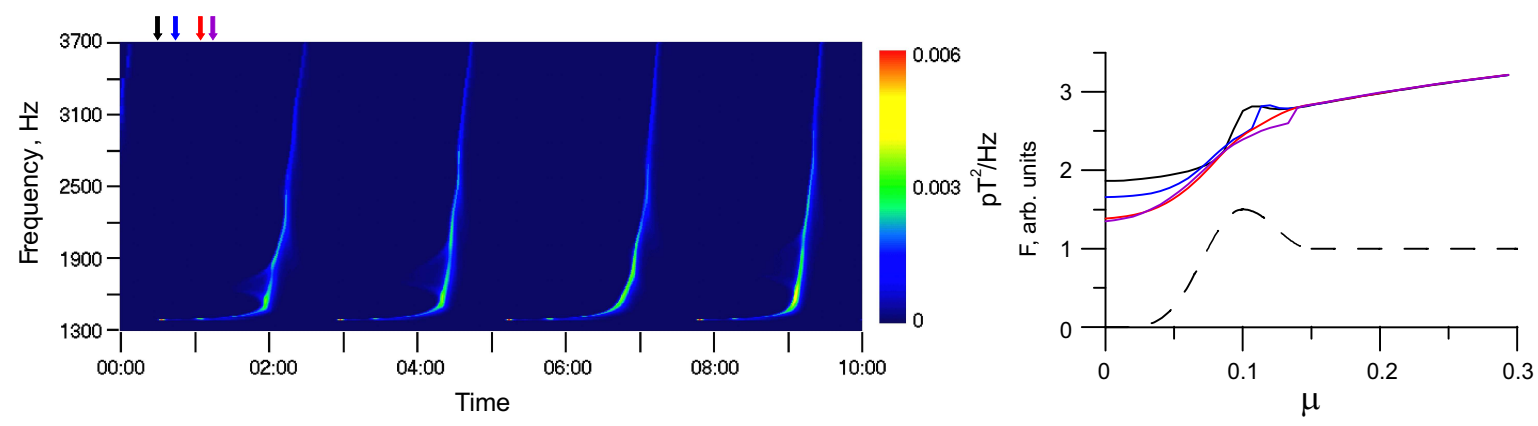

Fig. 6. Periodic generation of wave intensity spikes in the case of losses due to drift and nonmonotonic distribution (19) of energetic electrons in the the source; $\Delta=0.5, \delta_{1}=1, æ_{1}=0.05$, all other parameters as in Fig. 4.
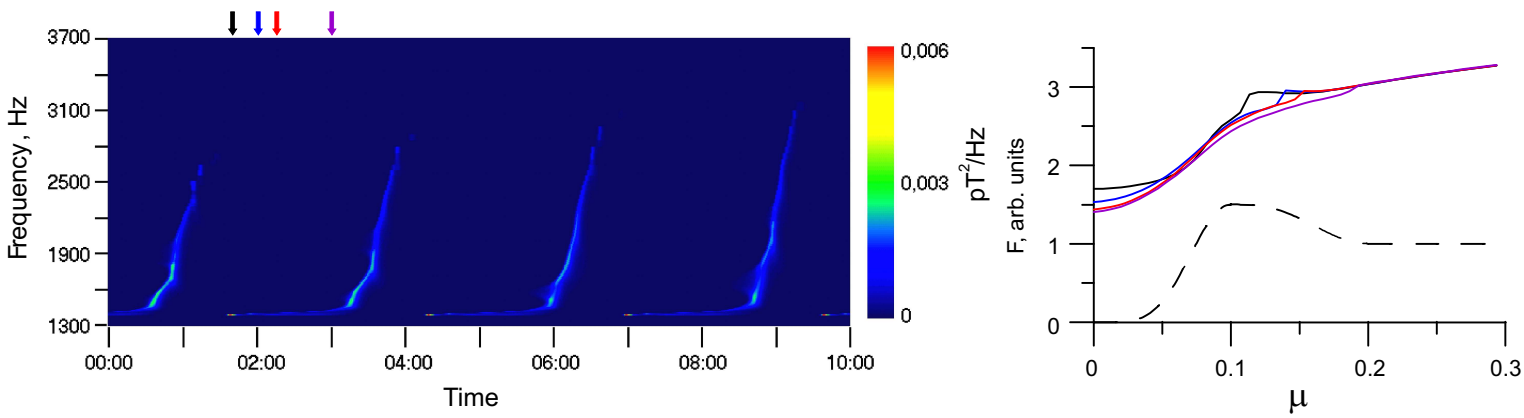

Fig. 7. The same as in Fig. 6 but for wider peak on the source pitch angle distribution: $\mathfrak{x}_{1}=0.1$. More intense and longer generation of low frequency waves at the beginning of the spike occurs.

not known. This problem requires special analysis, which is beyond the scope of this paper. To study the role of this system parameter, we consider two cases: an increase $(\ln R \sim \omega)$ and decrease ( $\ln R \sim \omega^{-1}$ ) of wave losses with frequency. With an increase in wave losses, the period of spike generation increases, because more time is needed to supply enough energetic particles to reach the instability threshold Eq. (16). The main difference between the cases of increasing and decreasing dependencies $R(\omega)$ is that in the latter case, the upper limit of generation frequency increases.

The third type of the source pitch angle distribution which we study in this paper is:

$$
\begin{aligned}
& F_{\text {in }}= \\
& \begin{cases}C \cdot[1+\Delta] \cdot \sin ^{\delta}\left(\frac{\pi}{2} \mathfrak{x} / \mathfrak{x}_{0}\right), & \mathfrak{x} \leq \mathfrak{x}_{0} \\
\left.C \cdot\left[1+\Delta-\Delta \cdot \sin ^{\delta_{1}}\left(\frac{\pi}{2}\left(\mathfrak{x}-\mathfrak{x}_{0}\right) / \mathfrak{x}_{1}\right)\right)\right], & \mathfrak{x}_{0}<\mathfrak{x} \leq \mathfrak{x}_{1}+\mathfrak{x}_{0} \\
C, & \mathfrak{x}>\mathfrak{x}_{1}+\mathfrak{x}_{0},\end{cases}
\end{aligned}
$$

with maximum at $\mathfrak{x}_{0}$; the parameter $\Delta$ characterizes the elevation of the maximum, while the parameters $\mathfrak{x}_{1}$ and $\delta_{1}$ characterize the width and steepness of the back slope. It is clear from Eq. (7) that the negative derivative of the distribution function $(\partial \Phi / \partial \mu<0)$ leads to wave damping, and this may give some interesting effects during wave generation.

The case of wide negative slope (i.e. large $\mathfrak{x}_{1}$ and values of $\delta_{1}$ which are not too high) is not very interesting. It may be considered as the case with monotonic source (Eq. (18)) and some additional effective damping term on the right-hand side of Eq. (2). Small values of $\Delta$ give almost no change to the generation regime. Extremely high $\Delta$ leads to very strong wave damping at high frequencies, so the generation is possible only in the low frequency band:

$\omega<\omega_{\max }=\omega_{B L} / \beta_{*} /\left(1-\mathfrak{x}_{0}\right)$.

The most interesting is the case of moderate $\Delta$ values and $\mathfrak{x}_{1} \sim \mathfrak{x}_{0}$. Three examples for $\Delta=0.5$ and different values of $\mathfrak{x}_{1}$ are shown in Figs. 6 to 8; all other parameters are the same as in Fig. 4. The first example (Fig. 6), corresponding to narrow peak at the source pitch angle distribution, is quite similar to the case of monotonic source (Fig. 4). The main difference is at the initial stage of a spike generation, when a wider frequency band is excited. The period of spike generation is slightly higher than in the case of Fig. 4, which is explained by the effective increase in wave energy losses (see the discussion above). With an increase in $\mathfrak{x}_{1}$, the generation of low frequency waves becomes more intense and takes longer time (Fig. 7). It is accompanied by a decrease in the maximum frequency of the generated waves and an increase in the generation period, which may be explained by an increase in effective wave losses. These generation regimes are qualitatively similar to the case of monotonic source (Eq. (18)). But for higher values of $\mathfrak{x}_{1}$, the generation regime changes (Fig. 8). As one can see, a persistent low frequency band with periodic modulation of its intensity 

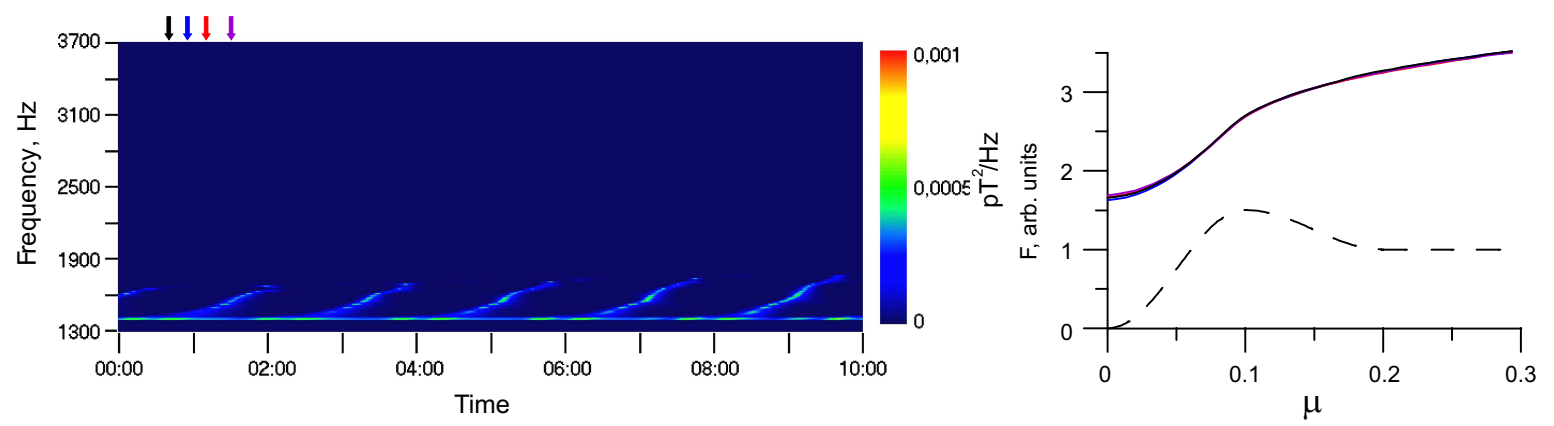

Fig. 8. The same as in Figs. 6 and 7 but for even wider peak on the source pitch angle distribution: $\mathfrak{x}_{1}=0.2$. Permanent generation in the low-frequency band is accompanied with periodic spike generation at higher frequencies.

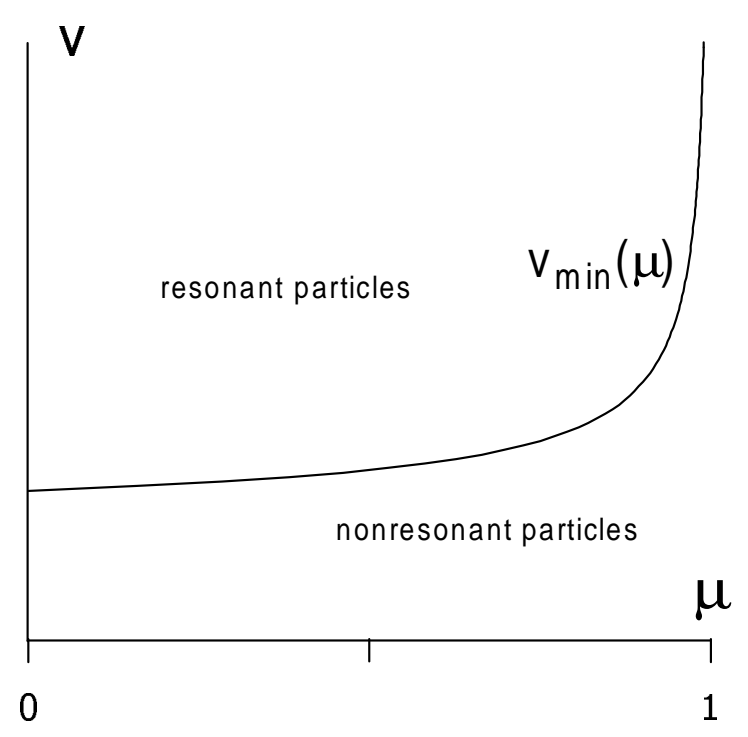

Fig. 9. The boundary between resonant and nonresonant regions for energetic electrons in the phase space.

and periodic spikes at higher frequencies is generated in this case. The period and the maximum frequency in the spike in this case are significantly smaller than in the case of smaller $\mathfrak{x}_{1}$ values.

\section{Conclusions}

Computer modeling of cyclotron instability in the Earth's magnetosphere in the framework of the self-consistent quasilinear theory reveals different regimes of ELF/VLF generation, including stationary generation, relaxation oscillations, and self-sustained oscillations of wave intensity. These regimes appear for both mechanisms of removal of energetic particles, i.e. due to the loss cone precipitation and due to the magnetic drift through the generation region. The main parameter determining the type of wave generation in the case of losses due to precipitation is the intensity of the energetic particle source $J_{0}$ (or, more exactly, the dimensionless intensity $j_{0}$ ). Relaxation oscillation of ELF/VLF wave inten- sity take place for small $J_{0}$ values. Relaxation oscillations characteristics, such as period and quality factor, constant shapes of wave spectrum and pitch angle distribution during a spike, are in good agreement with the analytical model in the two-level approximation, considered earlier by Bespalov and Trakhtengerts (1976). An increase in $J_{0}$ results in the transition to self-sustained oscillations of wave intensity with notable growth of wave frequency during a spike. The apparent cause of the self-sustained oscillations is involvement of new resonant electrons into the instability; this result is in agreement with previous studies by Bespalov and Trakhtengerts (1986) and Demekhov and Trakhtengerts (1994).

In the case of drift losses, the shape of energetic particle distribution in a source becomes more significant for the generation regime. In this case, a new type of dynamic spectra was obtained in computer modeling, which demonstrated alteration of spikes with constant and rising frequency (Fig. 5). It would be interesting to search such spectra in experimental data. In the case of drift removal, an important dynamical feature of an electron distribution function has been obtained-the formation of a step-like distortion, which, according to recent theoretical studies, can be a source of discrete ELF/VLF emissions (see in this relation Trakhtengerts, 1995, 1999).

\section{Appendix A}

The general expression for the kinetic growth rate of electromagnetic waves in a magnetized plasma due to cyclotron interaction is given by the following expression (Shafranov, 1967; Bespalov and Trakhtengerts, 1986):

$$
\gamma=\frac{4 \pi^{3}}{m} \sum_{s=-\infty}^{+\infty} \int G_{k, s}\left(\frac{s \omega_{B}}{\omega v_{\perp}} \frac{\partial}{\partial v_{\perp}}+\frac{k_{\|}}{\omega} \frac{\partial}{\partial v_{\|}}\right) f d^{3} v,
$$

where $\boldsymbol{k}$ is wave vector, $v_{\perp}$ and $v_{\|}$are the perpendicular and parallel components of the electron velocity with respect to the magnetic field, $f$ is electron distribution function; $G_{\boldsymbol{k}, s}$ determines the efficiency of cyclotron interaction of an electron with whistler wave on the $s$-th cyclotron harmonic. We refer the reader to the review by Bespalov and Trakhtengerts 
(1986) for the general expression of $G_{\boldsymbol{k}, s}$ for whistler waves. Other notations used in the Appendix are defined in Sect. 2.

In the case of waves propagating along the inhomogeneous geomagnetic field, the use of the local value of the growth rate (A1) for the analysis of wave generation is not quite correct. The one-hop amplification of a wave qualifying the growth of a wave amplitude on the entire path of the wave packet between conjugate ionospheres is required, which is defined as

$\Gamma(\omega, \boldsymbol{k})=\int_{-l}^{l} \frac{\gamma(\omega, \boldsymbol{k}, z)}{V_{g \|}} d z$

where $z$ is the coordinate along the magnetic field line (with the origin being at the equatorial plane), $\pm l$ are coordinates of the feet of the field line in the conjugate ionospheres, $V_{g \|}$ is the component of the group velocity parallel to the geomagnetic field.

For whistler waves propagating along the magnetic field $(\boldsymbol{k} \| \boldsymbol{B})$ only the interaction at the first cyclotron harmonic $(s=1)$ is possible, and in this case

$G_{k, 1} \equiv G_{k, 1}=\frac{e^{2} \omega V_{g}}{4 \pi c^{2} k} v_{\perp}^{2} \delta\left(\omega-k v_{\|}-\omega_{B}\right)$,

where $\delta$ is a Dirac delta function.

Using variables $\mu$ and $v$ the one-hop amplification for field-aligned whistler wave can be written as

$$
\begin{gathered}
\Gamma(\omega)=\frac{4 \pi^{4}}{m} \int_{-l}^{l} \int_{0}^{\infty} \int_{0}^{\omega_{B L} / \omega_{B}} \frac{G_{k, 1} \omega_{B}^{2}}{V_{g} \omega_{B L}^{2}} \times \\
\left(\frac{1}{v} \frac{\partial f}{\partial v}+\frac{2}{v^{2}}\left(\frac{\omega_{B L}}{\omega}-\mu\right) \frac{\partial f}{\partial \mu}\right) \frac{v^{2} d \mu d v d z}{\sqrt{1-\mu \omega_{B} / \omega_{B L}}} .
\end{gathered}
$$

After substitution of Eq. (A3) into this expression, we can remove the derivative $\partial f / \partial v$ by integrating this term by parts and obtain:

$$
\begin{aligned}
\Gamma(\omega)= & \frac{2 \pi^{3} e^{2}}{m c^{2} \omega_{B L}} \times \\
\int_{-l}^{l} \frac{\omega_{B}^{2}}{k} \int_{0}^{\infty} \int_{0}^{\omega_{B} / \omega_{B}}( & \left.\left(1-\frac{\omega}{\omega_{B}}\right) \mu \frac{\partial f}{\partial \mu}-\frac{\omega}{\omega_{B}} f\right) \times \\
& \frac{v^{2} \delta\left(\omega-k v_{\|}-\omega_{B}\right)}{\sqrt{1-\mu \omega_{B} / \omega_{B L}}} d \mu d v d z,
\end{aligned}
$$

where $v_{\|}=v \sqrt{1-\mu \omega_{B} / \omega_{B L}}$.

Changing the integration sequence and making integration over coordinate $z$ we obtain:

$$
\begin{aligned}
& \Gamma(\omega)=\frac{2 \pi^{3} e^{2}}{m c^{2} \omega_{B L}} \times \\
& \int_{0}^{1} \int_{v_{\min }}^{\infty}\left(\left(1-\frac{\omega}{\omega_{B L}}\right) \mu \frac{\partial f}{\partial \mu}-\frac{\omega}{\omega_{B L}} f\right) l_{\mathrm{eff}} v^{3} d v d \mu,
\end{aligned}
$$

where

$v_{\min }=\frac{\omega_{B L}-\omega}{k \sqrt{1-\mu}}$

is the boundary between resonant and nonresonant electrons (Fig. 9), which is determined from the cyclotron resonance condition at the equatorial plane, and

$l_{\mathrm{eff}}(k, \mu, v)=\left|\frac{\omega_{B}-\omega}{\omega_{B}^{2}} \frac{\partial}{\partial z}\left(\omega-k v_{\|}-\omega_{B}\right)\right|_{z=z_{\mathrm{res}}}^{-1}$

is the effective path of cyclotron interaction; here $z_{\text {res }}$ is the root of the equation $\omega-\omega_{B}\left(z_{\text {res }}\right)-k v \sqrt{1-\mu \omega_{B}\left(z_{\text {res }}\right) / \omega_{B L}}=0$.

The general expression for $l_{\mathrm{eff}}(k, \mu, v)$ is rather complicated. It can be written in a simple form if $\omega \ll \omega_{B L}$ and the parabolic approximation Eq. (10) for the magnetic field $B$ and cold plasma density distribution along the geomagnetic field line is used:

$l_{\mathrm{eff}} \approx\left|\frac{1}{\omega_{B}} \frac{\partial}{\partial z}\left(k v_{\|}+\omega_{B}\right)\right|_{z=z_{\mathrm{res}}}^{-1} \approx a \frac{1-\xi}{\sqrt{2-\xi}} \frac{1}{\sqrt{\xi-\mu}}$,

where $\xi(\omega, v)=1-\omega_{B L}^{2} /\left(k^{2} v^{2}\right)$. To obtain these expressions we also used the fact that the cyclotron resonance condition is fulfilled near the equatorial plane, i.e. $z_{\text {res }} / a \ll 1$.

Substituting Eq. (A9) in Eq. (A6) and neglecting small terms we obtain

$$
\begin{aligned}
\Gamma(\omega) & =\frac{2 \pi^{2} a \omega_{p L}^{2}}{\omega_{B L} c^{2} n_{c L}} \times \\
& \int_{0}^{1} \int_{\frac{\omega_{B L}}{k \sqrt{1-\mu}}}^{\infty} \frac{1-\xi}{\sqrt{2-\xi}}\left(\mu \frac{\partial f}{\partial \mu}-\frac{\omega}{\omega_{B L}} f\right) \frac{v^{2} d v d \mu}{\sqrt{\xi-\mu}} .
\end{aligned}
$$

And finally, to model the distribution with a narrow spread in energies we use the monoenergetic distribution function

$$
f(\mu, v, t)=\left(2 \pi v_{0}^{2}\right)^{-1} \delta\left(v-v_{0}\right) \tilde{f}(\mu, t) .
$$

Substituting this distribution in Eq. (A10) and taking the integral over velocity $v$ we obtain

$$
\begin{aligned}
\Gamma(\omega)= & \frac{\pi a \omega_{p L}^{2} v_{0}}{\omega_{B L} c^{2} n_{c L}} \times \\
& \frac{1-\xi}{\sqrt{2-\xi}} \int_{0}^{\xi}\left(\mu \frac{\partial \tilde{f}}{\partial \mu}-\frac{\omega}{\omega_{B L}} \tilde{f}\right) \frac{d \mu}{\sqrt{\xi-\mu}},
\end{aligned}
$$

where

$\xi \equiv \xi\left(\omega, v_{0}\right)=1-\left(\frac{\omega_{B L}}{k v_{0}}\right)^{2}=1-\frac{\omega_{0}}{\omega} ;$

here the following approximation for whistler wavevector $k=\omega_{p} / c \sqrt{\omega / \omega_{B}}$ is used, and $\omega_{0}$ is defined in Eq. (9).

Actually, Eq. (A13) is valid for any magnetic flux tube. In the case of the distribution function $\tilde{f}$, depending on the coordinate across geomagnetic field, we obtain the expression (7) after averaging Eq. (A13) over the interaction region. 
A similar technique is used to obtain Eq. (6) for diffusion coefficient. It includes averaging of the general expression (see Bespalov and Trakhtengerts (1986)) over the bounce oscillations of electrons and the use of approximation for $l_{\text {eff }}$ (A9).

Acknowledgements. This work was supported by Russian Foundation for Basic Research (grant No. 02-02-17109) and INTAS (grant No. 99-00502). D. L. Pasmanik and A. G. Demekhov was partially supported by the Commission on young scientists of the Russian Academy of Science.

Topical Editor T. Pulkkinen thanks two referees for their help in evaluating this paper.

\section{References}

Bespalov, P. A. and Trakhtengerts, V. Y.: The dynamics of the cyclotron instability in a magnetic trap, Fizika Plazmy, 2, 397-406, 1976.

Bespalov, P. A. and Trakhtengerts, V. Y.: The cyclotron instability in the Earth radiation belts, in: Reviews of Plasma Physics, edited by Leontovich, M. A., vol. 10, Plenum, New York, 155-192, 1986.

Davidson, G. T.: Self-modulated VLF wave-electron interactions in the magnetosphere: A cause of auroral pulsations, J. Geophys. Res., 84, 6517-6523, 1979.
Demekhov, A. G. and Trakhtengerts, V. Y.: A mechanism of formation of pulsating aurorae, J. Geophys. Res., 99, 5831-5841, 1994.

Hayakawa, M. and Sazhin, S. S.: Mid-latitude and plasmaspheric hiss: A review, Planet. Space Sci., 40, 1325-1338, 1992.

Hayakawa, M., Ohmi, N., Parrot, M., and Lefeuvre, F.: Direction finding of ELF hiss emissions in a detached plasma region of the magnetosphere, J. Geophys. Res., 91, 135-141, 1986.

Helliwell, R. A.: Whistlers and Related Ionospheric Phenomena, Stanford Univ. Press, Palo Alto, California, 1965.

Oguti, T., Hayashi, K., Yamamoto, T., Ishida, I., Higuchi, T., and Nishitani, T.,: Absence of hydromagnetic waves in the magnetospheric equatorial region conjugate with pulsating auroras, J. Geophys. Res., 96, 13 711-13 715, 1991.

Sato, N., Hayashi, K., Kokubun, S., Oguti, T., and Fukunishi, H.: Relationship between quasi-periodiv VLF-emission and geomagnetic pulsation, J. Atmos. Terr. Phys., 36, 1515-1526, 1974.

Sazhin, S. S. and Hayakawa, M.: Periodic and quasiperiodic VLF emissions, J. Atmos. Terr. Phys., 56, 735-753, 1994.

Shafranov, V. D.: Electromagnetic waves in plasma, in: Reviews of Plasma Physics, edited by Leontovich, M. A., vol. 3, 140, Consultants Bureau, New York, 1967.

Trakhtengerts, V. Y.: Magnetosphere cyclotron maser: Backward wave oscillator generation regime, J. Geophys. Res., 100, 17 205-17 210, 1995.

Trakhtengerts, V. Y.: A generation mechanism for chorus emission, Ann. Geophys., 17, 95-100, 1999. 